\title{
EFFET DE LA COMPLEMENTATION AVEC DES BLOCS MULTINUTRITIONNELS SUR LADEGRADABILITE DES FOURRAGES PAUVRES UTILISES DANS L'ALIMENTA- TION DES OVINS DU PLATEAU CENTRAL AU BURKINA
}

\author{
J. S. ZOUNDI ${ }^{1}$, A. J. NIANOGO ${ }^{2}$, L. SAWADOGO ${ }^{3}$
}

\begin{abstract}
${ }^{1} \mathrm{Dr}$ Jean Sibiri ZOUNDI* (Auteur-Corespondant), Institut de l'Environnement et de Recherches Agricoles (INERA), 04 BP 8645 Ouagadougou 04, BURKINA FASO ; Tel (226) 340270 ou 347112 ; Fax(226) 340271 ; e-mail : zoundi@hotmail.com ou zoundi@fasonet.bf
\end{abstract}

${ }^{2}$ Professeur Aimé Joseph NIANOGO, Université Polytechnique de Bobo, 01 BP 1091 Bobo-Dioulasso, BURKINAFASO ${ }^{3}$ Professeur Laya SAWADOGO, Unité de Formation et de Recherche Science, Vie et Terre (UFR-SVT),
Université de Ouagadougou, 03 BP 7021 Ouagadougou 03, BURKINA FASO

\section{RESUME}

Dans le but d'évaluer l'effet des blocs multinutritionnels sur l'utilisation fourrages pauvres (paille de sorgho (PS) et foin de Pennisetum pedicellatum, Trin.(Pp)), une étude de dégradation « in sacco » a été conduite sur des béliers munis de canules de rumen. Quatre traitements expérimentaux : T1, PS + bloc sans phosphore (BMN0); T2, PS + bloc avec phosphore (BMNP) ; T3, Pp + BMN0 ; T4, Pp + BMNP, ont été comparés à deux traitements témoins : l'un (T5) correspondant à des animaux utilisant une ration d'embouche intensive (40\% PS + $60 \%$ de concentré « Kibsa »), et l'autre correspondant à la situation des animaux utilisant les pâturages naturels en avril (T6), mai (T7) et en début juin (T8). La dégradation de la matière (MS) sèche présente des variations significatives $(\mathrm{P}<0,1 \%)$ avec les traitements : $57,9 \% ; 58,9 \% ; 62,8 \% ; 60,8 \% ; 59,0 \% ; 57,3 \% ; 45,5 \%$ et 53,0 \% pour le Pp à 96 h d'incubation, respectivement pour T1, T2, T3, T4, T5, T6, T7 et T8. La disparition de ADF et NDF suit les mêmes tendances ; les plus fortes valeurs étant enregistrées avec les traitements expérimentaux et T6. Ces variations seraient le fait de l'effet conjugué de la fourniture continue de $\mathrm{N}_{-} \mathrm{NH}_{3}$ et du niveau d'acidité dans le rumen. Il ressort de l'étude que les blocs, de par la fourniture continue de $\mathrm{N}-\mathrm{NH}_{3}$ et d'autres nutriments, permettent une amélioration significative de la dégradation des fourrages pauvres dans le rumen. Cela offre des possibilités pour leur valorisation en vue de l'utilisation optimale des fourrages pauvres pour la production de viande et l'entretien des femelles gestantes ou allaitantes en saison sèche chaude.

Mots-clés : dégradation « in sacco », fourrages pauvres, blocs multinutritionnels, N$\mathrm{NH}_{3}$ et $\mathrm{pH}$ du rumen, Burkina Faso 


\begin{abstract}
EFFECT OF MULTINUTRIENT BLOCKS SUPPLEMENTATION ON THE DEGRADABILITY OF POOR FORAGES USED FOR SHEEP FEEDING IN THE CENTRAL PLATEAU OF BURKINA FASO
\end{abstract}

With the goal of evaluating the effect of multinutrient blocks (MNB) on the use of poor roughages (sorghum straw (PS) and Pennisetum pedicellatum Trin. Hay (Pp)), « in sacco » degradation study was conducted with rams fitted with rumen cannulaes. Four experimental treatments: T1, PS + block without phosphorus (BMNO); T2, PS + block with phosphorus (BMNP); T3, Pp + BMNO; T4, $P p+B M N P$, were compared to two control treatments: one, (T5) corresponding to animals using an intensive fattening feed ( $40 \%$ PS $+60 \%$ concentrated « KIBSA ») and the other corresponding to the situation of animals using natural pasture in April (T6), May (T7), and early June (T8). The degradation of dry matter (MS) showed significant variations $(P<0.1 \%)$ with the treatments: $57.91 \%$; 58.8 \%; $62.8 \%$; 60.8 \%; $59.0 \%$; $57.3 \%$; $45.5 \%$ and $53.0 \%$ for Pp after 96 hours incubation, respectively for $T 1, T 2, T 3, T 4, T 5, T 6, T 7$, and T8. The disappearance of ADF and NDF followed the same pattern; the highest values were recorded with experimental treatments and T6. These variations may be due to the joint effect of the continuous supply of $\mathrm{N}-\mathrm{NH}_{3}$ and the level of acidity in the rumen. It is concluded that $\mathrm{MNB}$, because of their continuous supply of $\mathrm{N}-\mathrm{NH}_{3}$ and other nutrients, allow significant improvement of poor roughages degradability in the rumen. This offers possibilities for their valorization for meat production and for the maintenance of pregnant or lactating female during hot dry season.

Key words: « in sacco » degradation, poor roughages, multinutrient blocks, $\mathrm{N}$ $\mathrm{NH}_{3}$, and $\mathrm{pH}$ in the rumen, Burkina Faso

\section{INTRODUCTION}

L'alimentation des ruminants au sein des exploitations mixtes agricultureélevage des zones semi-arides en Afrique Sub-Saharienne repose essentiellement sur les pâturages naturels et les résidus de culture (Gryseels, 1988; „ ın ir. etal., 1992). Toutefois, plusieurs investigations (Penning De Vries et Djiteye, 1982 ; Savadogo, 2000) montrent qu'en dépit de leur abondance, les fourrages (pâturages naturels et les résidus de culture) disponibles en saison sèche sont très pauvres avec des taux de $\mathrm{N}$ généralement en dessous de $1 \%$. A cela s'ajoute le fait que la forte lignification de ceux-ci, de même que la nature de leurs constituants pariétaux, demeurent un sérieux handicap à leur bonne utilisation par les animaux (Bougouma-Yaméogo et al., 1996). Aussi, plusieurs méthodes ont-elles été développées ces dernières années en vue d'améliorer l'utilisation de tels fourrages, dont les traitements chimiques à l'urée ou à l'ammoniaque se sont montrés par ailleurs particulièrement efficaces (Bougouma-Yaméogo et al., 1996 ; Nianogo et al., 1997).

Concernant particulièrement l'engraissement saisonniers des ovins, un concentré « Kibsa » a été mis au point, avec une ration d'embouche intensive de $40 \%$ de paille plus $60 \%$ d'aliment « Kibsa » (Nianogo et al., 1995). Cette ration d'embouche permet une valorisation des fourrages pauvres, mais audelà de son coût de revient relativement élevé (plus de $450 \mathrm{~F} \mathrm{CFA/kg} \mathrm{de} \mathrm{gain),}$ l'accès du concentré au producteur pose également des difficultés.

D'autres investigations ont montré l'importance de l'efficacité de l'activité microbienne du rumen dans la dégradation des substrats pauvres (Thivend et al., 1985) ; laquelle est conditionnée par 
la disponibilité de certains éléments dont le $\mathrm{N}-\mathrm{NH}_{3}$, les peptides, de même que certains minéraux tels que le Soufre, le Magnésium, le Phosphore,... (Grenet et Besle, 1991 ; Ørskov, 1995). Ainsi, dans certains systèmes de production, de nombreux travaux ont montré que l'utilisation des blocs alimentaires contenant de l'urée constituait une alternative moins coûteuse permettant d'améliorer l'efficacité de la digestion des fourrages pauvres (El Fouly et Leng, 1987 ; Sansoucy, 1995 ; Toppo et al., 1997 ; Golluscio et al., 1998 ; Madhu et Singh, 1999).

Dans le plateau central du Burkina, l'alimentation des animaux en saison sèche apparaît très critique au vu des pertes énormes de poids de même que les mortalités enregistrées en cette période de l'année (Bicaba et al., 1986 ; Zoundi et al., 1994).

Dans le souci de développer des systèmes d'alimentation à moindre coût permettant d'atténuer ce stress nutritionnel en saison sèche et assurant aussi une utilisation optimale des fourrages pauvres pour les pratiques d'embouche, des blocs alimentaires ou multinutritionnels ont été fabriqués. Ceux-ci comprennent de l'urée et surtout des ingrédients facilement mobilisables par les producteurs tels que les sons de céréa- les, les fanes de légumineuses cultivées, les fruits de ligneux...

La présente étude se propose d'analyser les possibilités d'utilisation optimale de fourrages pauvres disponibles par le biais de la complémentation avec des blocs multinu-tritionnels. Elle examine plus particulièrement l'effet de la complémentation avec les blocs, en tant que substitut au concentré, sur la dégradation « in sacco » de fourrages pauvres disponibles en saison sèche.

\section{MATERIEL ET MÉTHODES}

\section{Blocs multinutritionnels}

Tenant compte de la nature des compléments alimentaires utilisés par les producteurs en saison sèche, les blocs multinutritionnels ont été fabriqués à partir d'ingrédients disponibles localement ou facilement accessibles (tableau 1). La composition moyenne des blocs est de : 95,1\% MS, 72,4\% MO, $27,9 \%$ NDF, 32,7 \% MAD et 0,56 UF/ $\mathrm{kg}$ MS. Cette formulation avait pour objectif majeur de fournir un environnement propice aux mico-organismes du rumen en vue d'une bonne dégradation des fourrages pauvres.

Tableau 1 : Composition des blocs multinutritionnels (en pourcentage)

$\mathrm{Pb}$ : Parkia biglobosa (Jacq.) Benth.

Composition of multinutrient blocks (percentage basis)

$\mathrm{Pb}$ : Parkia biglobosa (Jacq.) Benth.

\begin{tabular}{|c|c|c|c|c|c|c|c|c|c|}
\hline \multirow[t]{2}{*}{ Traitement } & \multicolumn{8}{|c|}{ Teneur en ingrédients } & \multirow[t]{2}{*}{ TOTAL } \\
\hline & Son de mil & Fanes d'arachide & $\begin{array}{l}\text { Gousses de } \\
\text { Piliostigma } \\
\text { reticulatum }\end{array}$ & $\begin{array}{c}\text { Poudre de néré } \\
(\mathrm{Pb})\end{array}$ & $\begin{array}{c}\text { Burkina } \\
\text { Phosphate }\end{array}$ & Urée & $\mathrm{NaCl}$ & $\overline{\text { Ciment }}$ & \\
\hline Bloc avec Phosphate brut & 35 & 5 & 10 & 20 & 5 & 10 & 5 & 10 & 100 \\
\hline Bloc sans Phosphate & 35 & 5 & 15 & 20 & 0 & 10 & 5 & 10 & 100 \\
\hline
\end{tabular}




\section{Dispositif expérimental}

L'essai a été conduit avec neuf (09) béliers Bali Bali munis de canules de rumen et de poids vif variant entre 45 et $48 \mathrm{~kg}$.

Quatre rations expérimentales ont été constituées à partir de deux fourrages de base de qualité différente : la paille de sorgho (PS) $(0,30 \%$ MAD ; $0,30 \mathrm{UF} / \mathrm{kg} \mathrm{MS}$ ) et le foin de Pennisetum pedicellatum Trin. (Pp) (2,90\% MAD ; 0,39 UF/kg MS).

* Ration 1 (T1) : PS ad libitum + bloc multinutrionnel sans phosphate (BMNO)

* Ration 2 (T2) : PS ad libitum + bloc multinutrionnel incorporant du phosphate brut (BMNP) BMNO

* Ration 3 (T3) : Pp ad libitum + BMNP

* Ration 4 (T4) : Pp ad libitum +

Tenant compte de l'objectif de l'étude qui est d'analyser les possibilités d'utilisation des blocs alimentaires en tant qu'alternative moins coûteuse de substitut aux concentrés, permettant une valorisation des fourrages pauvres pour les pratiques d'engraissement des animaux ou par les animaux utilisant les pâturages naturels, les rations expérimentales ont été comparées à deux témoins. Le témoin 1 (T5) correspond à une ration d'embouche intensive : $40 \%$ PS + $60 \%$ de concentré « Kibsa » (KSA) de valeur 10,68 \% MAD et 0,67 UF/kg MS. Le témoin 2 correspond à la situation où les animaux exploitent des parcours naturels à Pennisetum pedicellatum, Andropogon pseudapricus Stapf., Andropogon gayanus Kunth., Pilostigma reticulatum (DC.) Hochst, Guiera sengalensis J.F. Gmel., Ziziphus mauritiana Lam., Balanites aegyptiaca (Linn.) Del., pendant les périodes jugées les plus critiques de l'année (Bicaba et al., 1986) : avril (T6), mai (T7) et début juin (T8). Les animaux bénéficient de 8 heures de pâture journalière.

La paille de sorgho $(90,8 \% \mathrm{MO}$, $40,3 \%$ CB, 47,9 \% ADF, 7,3\% ADL, $76,0 \%$ NDF et 3,7 MAT) et le foin de Pennisetum pedicellatum (90,2\% MO, $34,5 \%$ CB, 54,0 \% ADF, 11,6\% ADL, 81,1\% NDF et 5,0\% MAT) ont été utilisés comme fourrage pour la dégradabilité « in sacco ». Ces aliments ont été broyés à des dimensions d'environ $2 \mathrm{~mm}$. Pour chaque animal et pour chaque traitement, 5 sachets (3-5 g d'aliment par sachet de $11 \mathrm{~cm} \times 7 \mathrm{~cm}$ et de 20 microns de porosité) ont été préparés par type de fourrage, dont un sachet est lavé au temps zéro pour la détermination de la fraction immédiatement soluble. Quatre temps d'incubation (24, 48, 72 et 96 h) ont été retenus et le nombre total de sachets introduits par traitement et par type de fourrage est de : 4 sachets $\times 9$ animaux $\times 3$ fois. Après leur retrait du rumen, les sachets sont rincés à l'eau pendant 20 min et portés ensuite à l'étuve $\left(65^{\circ} \mathrm{C}\right)$ pendant $72 \mathrm{~h}$ pour la détermination de la matière sèche (MS). Le dosage de la matière organique (MO), des fibres détergents acides (ADF) et de fibres détergents neutres (NDF) a été fait selon les procédés de AOAC (Aoac, 1995).

Les paramètres de dégradabilité ont été déterminés à l'aide de l'équation $y=a+b(1-e-c t) d e$ Ørskov et McDonald (1979) et en utilisant les procédures NLIN de SAS (Sas, 1990) : y, étant la fraction effectivement disparue au tempst ; a, la fraction immédiatement soluble; b, la fraction progressivement dégradable et $c$, la vitesse de dégradabilité de la fraction lentement dégradable. La dégradabilité théorique (Dt) a été calculée à l'aide de l'équation 
(Ørskov et McDonald, 1979 ; MichaletDoreau et al., 1987), Dt (\%) = a + (bc)/ $(\mathrm{c}+\mathrm{k}), \mathrm{k}$ étant la vitesse de transit des particules hors du rumen (en h-1).

Pour tous les traitements, des prélèvements de jus de rumen ont été effectués et les dosages de $\mathrm{N}-\mathrm{NH}_{3}$ faits selon la méthode CONWAY (Conway, 1962). Pour chaque prélèvement, deux mesures sont faites : le matin à $10 \mathrm{H} 00$ et l'après-midi à $17 \mathrm{H} 00$. Pour le témoin utilisant la ration d'embouche intensive, les mesures sont faites chaque fois 3 heures après la fourniture du KSA. Des analyses de variance (ANOVA) ont été effectuées sur les valeurs de dégradabilité en utilisant les procédures GLM de SAS (Sas, 1990). La comparaison des moyennes des différents traitements a été faite selon le test de Student Newman-Keuls. Concernant particulièrement les traitements expérimentaux une analyse fine a été faite en vue d'apprécier l'effet des deux facteurs principaux : le type de fourrage de base (PS, Pp), le phosphore contenu dans les blocs.

\section{RESULTATS}

\section{Consommation des blocs et profil fermentaire du rumen}

Les résultats obtenus sur la consommation de blocs avec les traitements expérimentaux (tableau 2) montrent des variations hautement significatives $(P<0,01 \%)$ entre les traitements expérimentaux: 127,4 g, 57,9 g, 336, 7 $\mathrm{g}$ et $117,4 \mathrm{~g}$, respectivement pour $\mathrm{T} 1$, T2, T3 et T4.

Les niveaux de $\mathrm{N}-\mathrm{NH}_{3}$ (tableau 2) montrent également des variations significatives $(P<0,01 \%)$ avec les traite- ments : 127,7 mg/l, 99,2 mg/l, 228,9 mg/ I, 146,7 mg/l, 184 mg/l, 164 mg/l, 208,7 $\mathrm{mg} / \mathrm{l}$ et $175 \mathrm{mg} / \mathrm{l}$, respectivement pour T1, T2, T3, T4, T5, T6, T7 et T8.

Les variations observées avec le $\mathrm{pH}$ sont également significatives $(P<0,01 \%)$ avec les traitements; les plus faibles valeurs étant enregistrées avec les animaux utilisant les parcours naturels en avril (T6), mai (T7) et début juin (T8) : 6,$38 ; 6,38$ et 6,40 respectivement.

Dégradation de la matière sèche, de la matière organique et des constituants des parois cellulaires

La disparition de la matière sèche (MS) dans le rumen (tableau 2) présente des variations significatives ( $P<0,01 \%)$. Pour PS à $96 \mathrm{~h}$ d'incubation, les valeurs enregistrées sont de : $55,5 \%, 57,2 \%$, $53,6 \%, 57,4 \%, 52,7 \%, 57,3 \%, 50,4$ $\%, 52,0 \%$, respectivement pour T1, T2, T3, T4, T5, T6, T7 et T8. Pour Pp à $96 \mathrm{~h}$ d'incubation, les valeurs enregistrées sont de : $57,9 \%, 58,8 \%, 62,8 \%, 60,8$ $\%, 59,0 \%, 57,3 \%, 46,0 \%$ et $53,0 \%$, respectivement pour T1, T2, T3, T4, T5, T6, T7 et T8.

Exception faite de PS à $96 \mathrm{~h}$ d'incubation, la dégradation de la matière organique (MO) présente également des variations hautement significatives $(P<0,01 \%)$ avec les traitements. Ces mêmes variations significatives sont observées (tableau 3) avec la dégradation des constituants des parois cellulaires (ADF, NDF).

D’une manière générale et quel que soit le fourrage incubé, les plus fortes disparitions sont observées avec les traitements expérimentaux T2, T3 et T4, et avec les animaux utilisant le pâturage naturel en avril (T6). Les plus faibles valeurs quant à elles sont enregistrées 
avec les animaux utilisant les pâturages naturels en mai (T7) et en début juin (T8). L'analyse globale des résultats montre qu'à quelques exceptions près, les résultats obtenus sur la disparition de NDF pour PS et $P p$ à $96 \mathrm{~h}$ avec les traitements expérimentaux sont supérieurs à ceux du témoin utilisant les pâturages naturels (T6, T7 et T8).

Les paramètres de dégradabilité (tableaux 4 et 5) montrent les mêmes tendances que celles observées avec la dégradation de la MS ; les valeurs les plus élevées en terme de dégradabilité théorique pour MS, MO, ADF et NDF étant à l'avantage des traitements expérimentaux quel que soit le type de fourrage ( $P S$ ou $P p)$.

Les résultats obtenus (tableaux 2 et 3) montrent également un effet positif du fourrage de base utilisé pour l'alimentation (PS ou Pp). Dans la plupart des cas où l'effet « fourrage de base » est significatif, on a le foin de Pp qui donne des résultats supérieurs à la paille de sorgho (avec des augmentations moyennes de dégradation de 4 points).

L'effet du phosphore (tableaux 2 et 3) quant à lui est variable selon la nature du produit mis en incubation. Avec la paille de sorgho, on a toujours un effet positif dû à l'apport de phosphore (augmentation moyenne de la dégradation de 5 et 3 points, respectivement pour ADF et NDF) et ce malgré les faibles consommations enregistrées avec ce type de bloc. Dans le cas où le fourrage incubé est de qualité relativement moyenne (le foin de $P$. pedicellatum), les plus fortes valeurs de dégradabilité sont enregistrées dans la plupart des cas avec la formule BMNO (augmentation moyenne de 6 et 4 points, respectivement pour ADF et NDF).

Tableau 2: Consommation de blocs alimentaires, Caractéristiques du milieu rumenal et dégradabilité (en \%) de la matière sèche (MS) et de la matière organique (MO).

NB : a) PS = Paille de sorgho $; \mathrm{Pp}=$ Pennisetum pedicellatum $; \mathrm{BMNO}=$ Bloc multinutritionnel sans phosphate ; BMNP = Bloc multinutritionnel avec le phosphate ; KSA = Concentré " Kibsa " ; PN = Pâturage naturel

b) Les valeurs figurant sur la même ligne et ne portant aucun indice commun son significativement différentes pour les niveaux de significations suivants : ** $=\mathrm{P}<0,01 \% ; * \star=$ $\mathrm{P}<0,1 \% ;{ }^{*}=\mathrm{P}<5 \%$ selon le test de Student-Newman-Keuls ; NS = Non significatif

Blocks intake, ruminal caracteristics, and dry matter (DM) and organic matter(OM) degradability

NB : a) $P S=$ sorghum straw $; P p=$ Pennisetum pedicelatum ; BMNO = Multinutrient block without phosphate ; BMNP = Multinutrient block with phosphate ; KSA = Concentrate "Kibsa " $P N=$ Natural pasture

b) Means in the same row with different labels are significantly different for the following levels of significance : ${ }^{* *}=P<0,01 \% ; * \star=P<0,1 \% ; *=P<5 \%$ according to StudentNewman-Keuls Test; NS = Non significant 


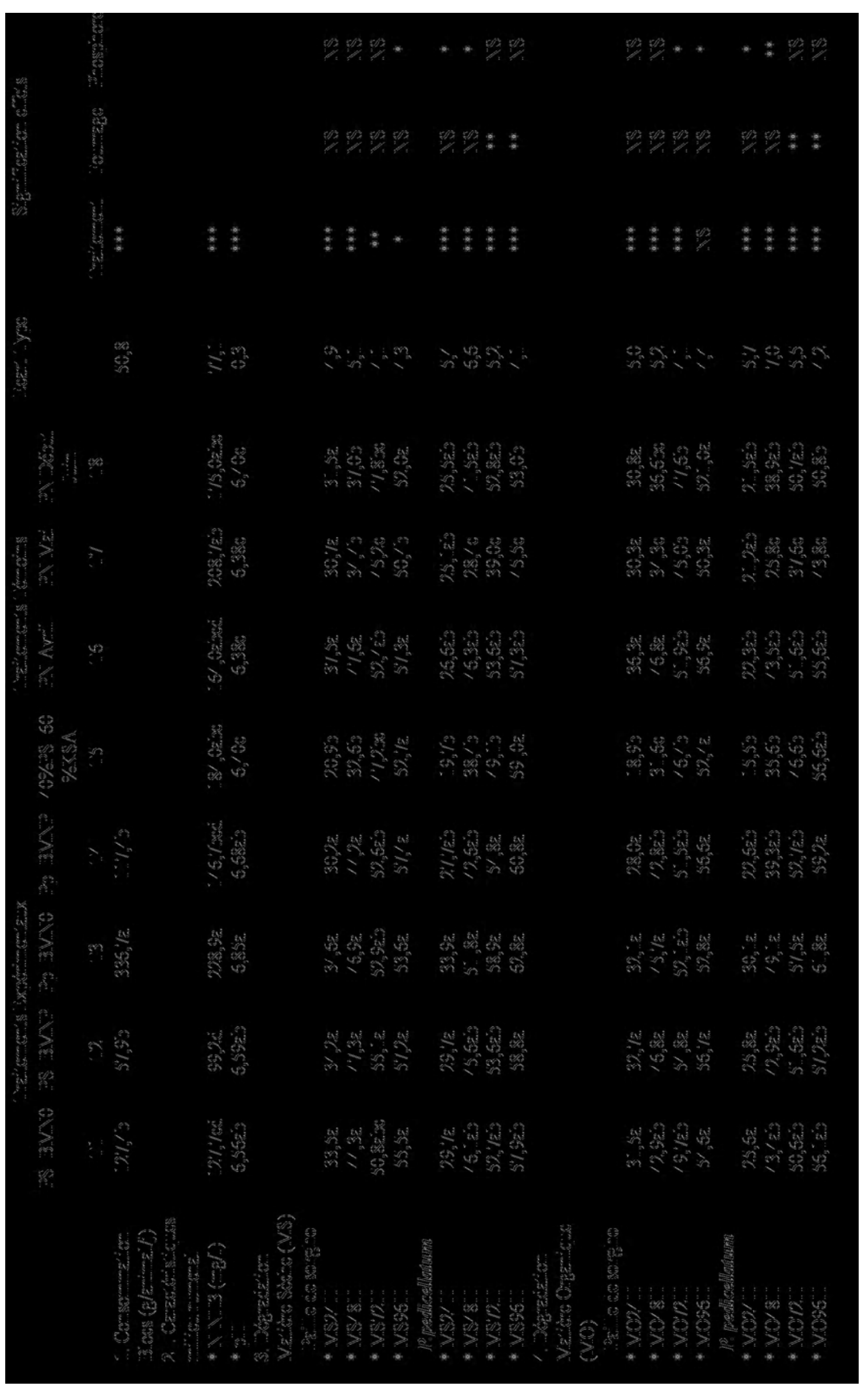

Agronomie Africaine 15 (2) : 77-92 (2003) 


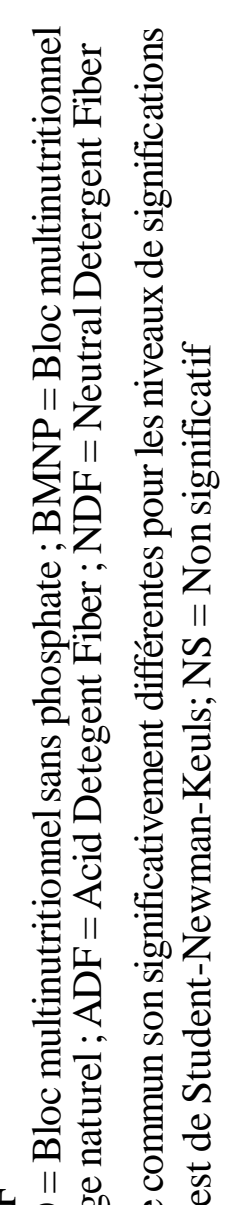

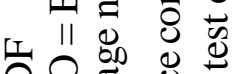

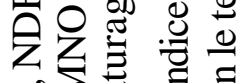
地范

\&.a

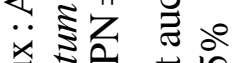

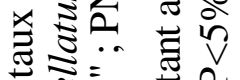

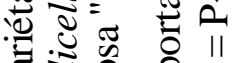

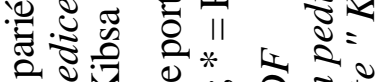

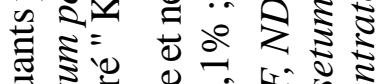

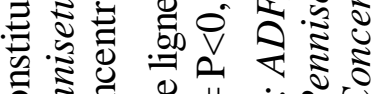

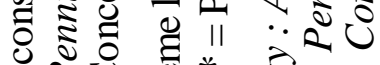
थ

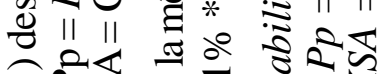
จำ ฮี่

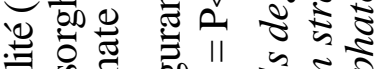

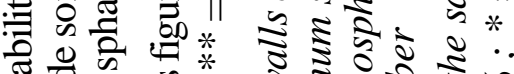
त्ठ

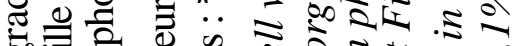

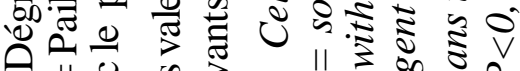

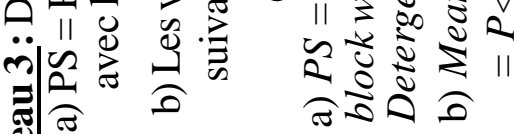
정

畆苔 $\stackrel{*}{*}$

do

o

a

*

$\because$

ప

:

$\frac{\pi}{4}$

¿

$\frac{n}{2}$

बे

श은

을.

요

운

का 11

$\frac{2}{2}$

केँّ

i)

齐

ป

हิ

के

ธิ

$\frac{0}{2}$

ธิ

皮

एक

ปี

忥

วิ

ㄷำ

\& 2

है 11

*

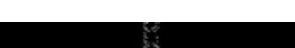

$4: 20$

.

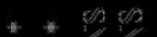

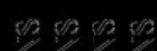

管:

答留的

- : : :

*: : :

$\div \div \div$

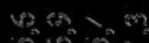

केषिए

पर

ำ

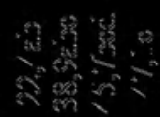

然遥的

背 5

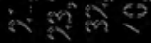

吩然

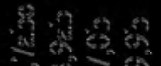

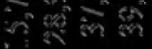

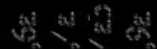

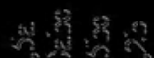

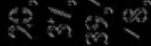

踏的

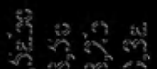

कर सह की

繁繁照

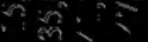

然然

的然的

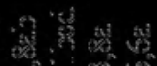

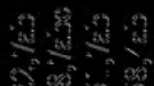

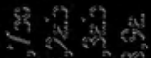

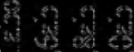

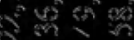

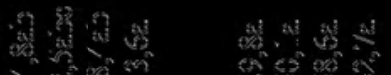

की

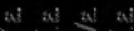

的照

给然然

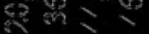

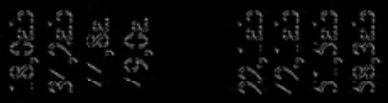

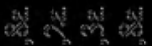

象的

今留然话

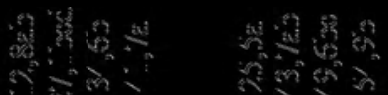

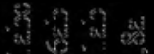

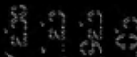

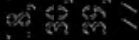




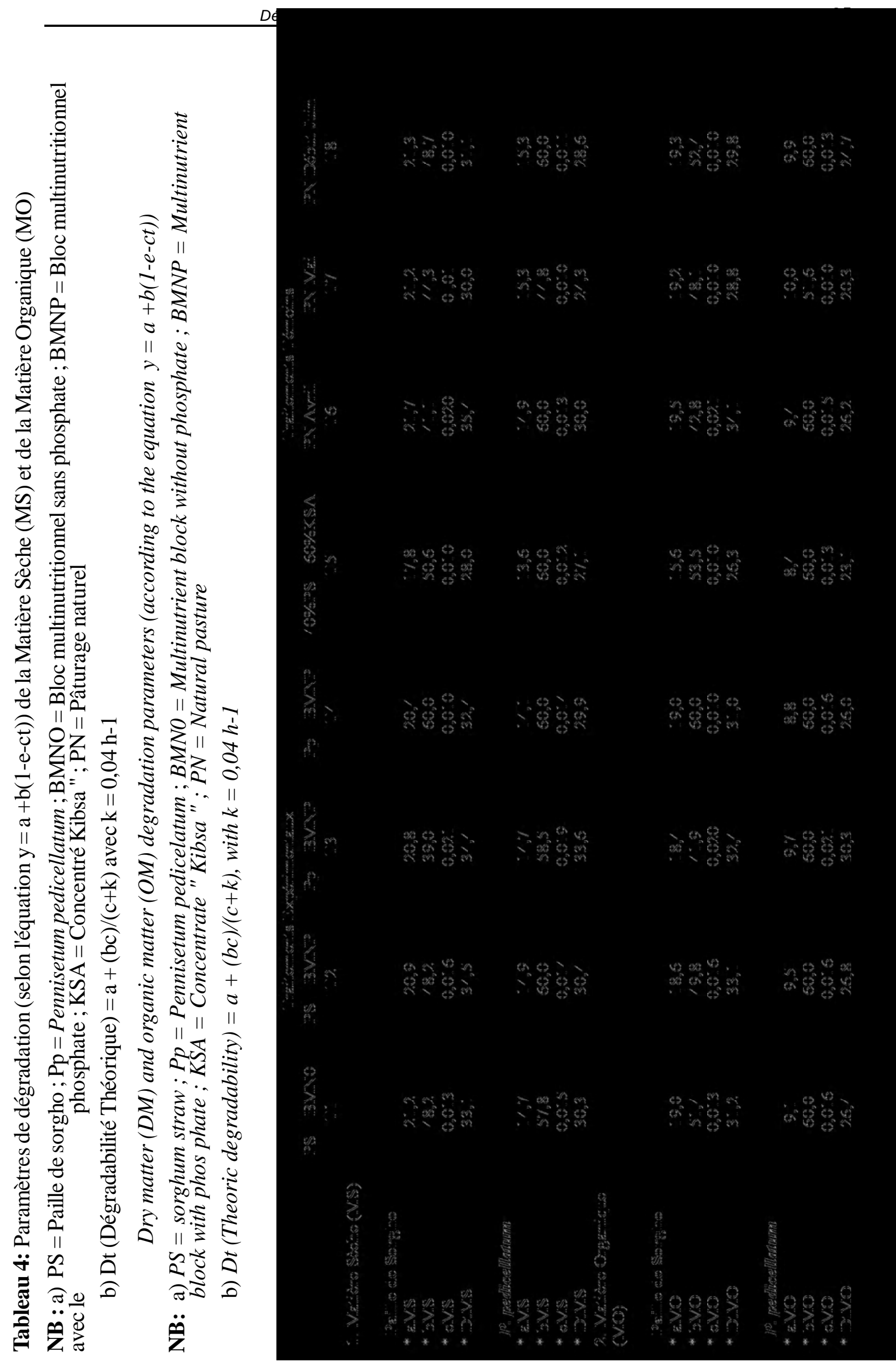




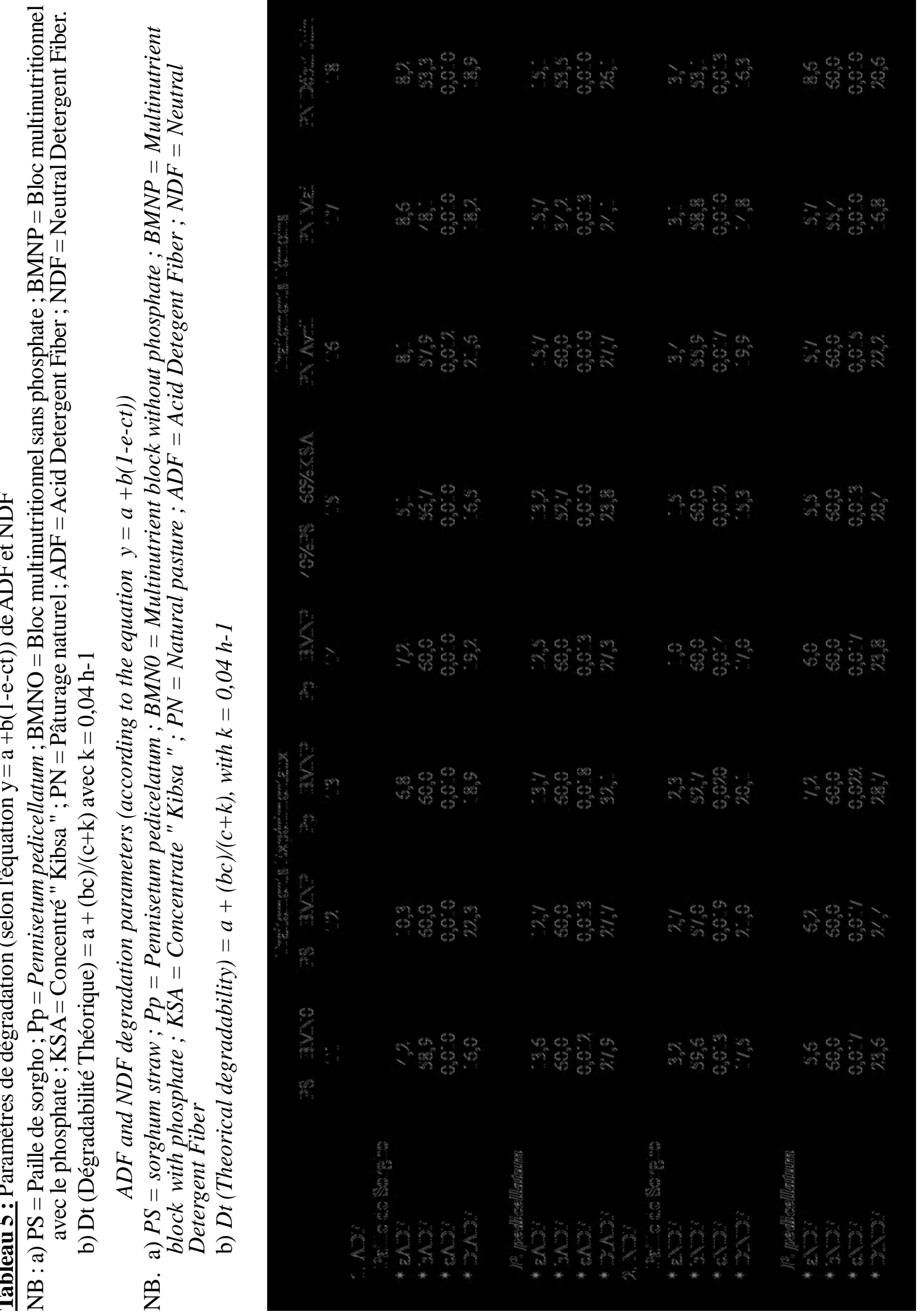




\section{DISCUSSIONS}

\section{Consommation de blocs et niveaux de $\mathrm{N}-\mathrm{NH} 3$ et $\mathrm{pH}$}

Les résultats observés pour la consommation des blocs sont comparables à ceux enregistrés par El Fouly et Leng (1987). Ils montrent également que les plus faibles consommations sont enregistrées avec les blocs incorporant le phosphore et cela traduirait une réaction particulière des animaux par rapport à la présence de certains minéraux dans les blocs. El Fouly et Leng (1987) ont observé que la présence de $\mathrm{S}$ et de $\mathrm{K}$ stimulait par contre la consommation de blocs. D'une manière générale, les résultats de la consommation ont été sujets à de très fortes variabilités, avec des consommations minimales voisines de zéro pour tous les traitements. Cette même tendance a été observée par d'autres auteurs (El Fouly et Leng, 1987) et serait liée à certains facteurs tels que la durée de la complémentation, le degré de solidification des blocs et la présence de certains minéraux. Elle pourrait également traduire les différences de comportement individuel entre les animaux en matière de consommation des blocs.

Exception faite du traitement 2 (PS + BMNP), les niveaux de $\mathrm{N}-\mathrm{NH}_{3}$ enregistrés avec les traitements expérimentaux sont comparables avec ceux des témoins. Les résultats obtenus avec les traitements expérimentaux (hormis ceux du traitement 2) sont légèrement inférieurs aux valeurs (160-228 mg/l) enregistrées par Rueda et De Combellas (1999) avec une consommation moyenne de blocs de 192,80 g/animal. Les valeurs enregistrées avec les animaux utilisant les parcours naturels sont particulièrement très élevées et supé- rieures à celles obtenues par Sawadogo et al. (1995) avec des ovins Djallonké à cette même époque de l'année. Dans le cas de notre étude, des ovins de race Bali Bali, animaux plus habitués à exploiter des fourrages aériens (ligneux) riches en azote, ont été utilisés et les fortes valeurs observées seraient liées au type d'animal utilisé.

En dehors de la valeur moyenne enregistrée avec T2, les niveaux de N$\mathrm{NH}_{3}$ enregistrés atteignent les valeurs optimales $(150 \mathrm{mg} / \mathrm{l})$ requises pour une bonne dégradation des fourrages pauvres (Preston, 1986).

\section{Dégradation de la Matière Sèche, de la Matière Organique et des consti- tuants des parois cellulaires}

Les valeurs de dégradation de la MS obtenues à $24 \mathrm{~h}$ d'incubation avec les deux fourrages (PS et Pp) sont inférieures à celles enregistrées par Ouédraogo (1998) avec la paille de blé sur des rations à base de foin de luzerne/ dactyle et de foin de prairie. Les valeurs obtenues avec les traitements expérimentaux à $48 \mathrm{~h}$ sont cependant supérieures à celles enregistrées par Nandra et al. (1993) et Yaméogo et al. (1991) avec la paille de blé. Exception faite du pâturage naturel en avril (T6), les résultats obtenus avec les traitements expérimentaux sont dans la plupart des cas supérieurs à ceux enregistrés avec les témoins.

Les résultats enregistrés pour le NDF du foin de $P$. pedicellatum avec les traitements expérimentaux à $48 \mathrm{~h}$ d'incubation sont comparables à celles (variant de 41,3 à 45,5\%) enregistrées par Oosting et Waanders (1993) avec la paille de blé.

Les valeurs de dégradabilité théorique (Dt) enregistrées pour la MS sont 
dans la fourchette des valeurs $(20,80$ $42,60 \%)$ obtenues par BougoumaYaméogo et al. (1996) avec des graminées de pâturage naturel et des résidus de culture sur des animaux consommant une ration à $13 \%$ de MAT. Les valeurs de Dt pour la NDF de la paille de sorgho sont également comparables à celles enregistrées par Ranilla et al. (1997) avec la paille de blé et sur des animaux nourris au foin de luzerne. Comme pour la dégradation de la MS, on constate que les plus faibles valeurs de Dt sont dans la plupart des cas enregistrées avec les traitements 5, 7 et 8 .

Toutes ces différences observées entre les traitements en matière de dégradation de la MS, MO et des constituants pariétaux (ADF, NDF) et de dégradabilité théorique, s'expliqueraient en grande partie par les conditions environnementales du rumen (disponibilité en $\mathrm{N}-\mathrm{NH}_{3}, \mathrm{pH}$ ). Au delà des valeurs moyennes calculées de $\mathrm{N}-\mathrm{NH}_{3}$, les différences de dégradation observées entre les animaux utilisant la ration d'embouche (T5) et ceux exploitant les pâturages naturels en mai (T7) et début juin (T8), et les rations expérimentales ( $T 1$, T2 et T4), s'expliqueraient par le fait que la consommation de blocs permet d'avoir une bonne disponibilité en $\mathrm{N}-\mathrm{NH}_{3}$ de façon continue dans le rumen. Plusieurs travaux (Preston, 1986 ; El Fouly et Leng, 1987) ont mis en évidence l'importance d'un tel paramètre sur l'action des micro-organismes. Il apparaît donc qu'une augmentation ponctuelle de $\mathrm{N}$ $\mathrm{NH}_{3}$, aussi importante soit-elle, a moins d'effets, comparée à une production moyenne mais continue de ce nutriment. Cela pourrait expliquer les résultats enregistrés avec le témoin utilisant la ration d'embouche (T5), situation similaire à celle observée par Urbaniak (1995). Aussi, la supériorité des traitements ex- périmentaux en matière de dégradation de l'ADF et du NDF, comparativement aux animaux utilisant la ration d'embouche (T5) indiquerait qu'au-delà du N-NH${ }_{3}$, la fourniture de $\mathrm{N}$-protéique à travers les blocs a eu un effet sur l'activité microbienne. Cela serait en concordance avec les travaux de Carro et Miller (1999) qui ont mis en évidence l'importance d'autres formes de N sur la digestion optimale des fibres dans le rumen.

Au delà de la fourniture en $\mathrm{N}-\mathrm{NH}_{3}$ et en N-Protéique, les variations observées pourraient également s'expliquer par les niveaux de $\mathrm{pH}$. En effet, les plus faibles valeurs ( $\mathrm{pH} 5$ ) ont été enregistrés avec les traitements 7 et 8 et pourraient avoir influencé négativement la dégradation des fibres et de la MS, comme cela a déjà été mis en évidence par de nombreux auteurs (Gangwar et al. , 1998 ; Stritzler et al., 1998, MansoAlonso et al., 1999 ; Olson et al., 1999 ; Carro et al ., 2000). Bien que l'augmentation des niveaux de $\mathrm{N}-\mathrm{NH}_{3}$ ait une influence positive sur la dégradation des substrats pauvres (Kanjanapruthipong et Leng, 1998 ; Dixon, 1999 ; Singh et al., 1999), les $\mathrm{pH}$ faibles nuiraient fortement à l'activité microbienne. Cela expliquerait en partie les faibles valeurs de dégradation obtenues avec le témoin en mai (T7) et en début juin (T8) en dépit des fortes moyennes de $\mathrm{N}-\mathrm{NH}_{3}$ enregistrées. Certains auteurs (Veth et al., 1999) ont enregistré des baisses de 14 et $11 \%$ de la digestion de la MS et du NDF avec une diminution du $\mathrm{pH}$ de 6,6 à 5,4 .

L'effet du phosphore sur la dégradation des fourrages est variable selon le type de substrat incubé. Ainsi, les plus fortes valeurs de dégradabilité observées pour Pp avec les blocs sans phosphate comparativement à ceux contenant du phosphate, indiqueraient un 
effet probable des fortes productions de $\mathrm{N}-\mathrm{NH}_{3}$ due à la bonne consommation de BMNO comparativement aux blocs incorporant du phosphore (BMNP).

Quant à la dégradation de PS, les effets positifs enregistrés avec l'apport de phosphore dans les blocs, traduisent l'importance de ce minéral pour l'activité microbienne du rumen. Ces résultats rejoignent ceux d'autres auteurs (Komisarczuk-Bony et Durand, 1991) qui ont mis en évidence le fait que ce minéral est spécialement requis pour la dégradation des fourrages pauvres et plus particulièrement des constituants des parois cellulaires (notamment celle de la cellulose). Ces tendances confirment le fait qu'au delà des faibles teneurs en azote, les fourrages disponibles présentent des déficiences pour ce minéral.

Aussi, l'effet positif du fourrage de base utilisé dans l'alimentation traduit la différence de valeur nutritive entre les deux fourrages utilisés (PS et Pp). Ces résultats indiquent également que l'effet des blocs pourrait être plus perceptible si le fourrage de base utilisé pour l'alimentation est de bonne valeur nutritive.

\section{CONCLUSION}

La fourniture continue de $\mathrm{N}-\mathrm{NH}_{3}$ et d'autres nutriments azotées et énergétiques à travers l'utilisation des blocs multinutritionnels seuls en complémentation de régimes extrêmement pauvres, permet d'assurer des niveaux de dégradabilité de la paille de sorgho et du foin de $P$. pedicellatum comparables à ceux offerts par la ration d'embouche intensive et supérieurs aux valeurs enregistrées avec les animaux utilisant les parcours naturels en mai et en début juin. Au delà de la variabilité ob- servée au niveau des effets, l'incorporation du phosphore dans les blocs revêt toute son importance dans le cadre de la valorisation des fourrages pauvres.

Les résultats obtenus offrent des perspectives intéressantes pour une valorisation des blocs en combinaison avec d'autres sources énergétiques et protéiques en vue de l'utilisation optimale des fourrages pauvres. De même, et au vu de l'importance de la fourniture continue de $\mathrm{N}-\mathrm{NH}_{3}$ dans le rumen, l'utilisation des blocs pendant la période critique de saison sèche chaude constituerait une voie intéressante aussi bien pour les animaux à l'entretien que pour le femelles gestantes ou allaitantes.

\section{REFERENCES}

ANONYME 1, 1995 : Association of Official Analytical Chemist (AOAC). 1995. Official methods of analysis. 17th edition, AOAC, Washington, DC, USA, $2000 \mathrm{p}$.

BICABA (Z.M.), (R.) BOSMA, (G.) MACON, (M.) SABA, (S.J.) ZOUNDI. 1986. Etude de la croissance compensatrice chez les zébus au Burkina : Analyse comparative après divers degrés de sous-nutri-tion.Rev.Elev. Méd. pays trop. 39 (3-4), 414-420.

BOUGOUMA-YAMEOGO (V.), (R.) CORDESSE, (A.J.) NIANOGO, (M.) INESTA, (S.) NASSA. 1996. Modifications chimiques et estimation de la dégradabilité de la matière sèche de quatre fourrages tropicaux traités à l'ammoniac ou à l'urée. Revue Méd. Vét. 147 (10), 689-700.

CARRO (M.D.), (E.L.) MILLER. 1999. Effect of supplementing a fibre basal diet with different nitrogen forms on ruminal fermentation and microbial growth in an in vitro semicontinuous culture system (RUSITEC). British Journal of Nutrition 82 (2), 149-157.

CARRO (M.D.), (C.) VALDES, (M.J.) RANILLA, (J.S.) GONZALEZ. 2000. Effect of forage to concentrate ratio in the diet on ruminal fermentation and digesta flow kinetics in sheep offered food at a fixed and restricted level of intake. Animal Science 70 , 127-134. 
CONWAY (E.J.) 1962. Microdiffusion analysis and volumetric error. 5th Edition. Crosby Lockwood, London (UK), 468 p.

DIXON (R.M.). 1999. Effect of addition of urea to a low nitrogen diet on the rumen digestion of a range of roughages.Australian Journal of Agricultural Research 50 ( 6), 1091-1097.

EL FOULY (H.A.), (R.A.) LENG, 1987. Enhancing fermentative digestion of cereal straws by using urea-molasses multinutrient bolck. In IAEA (Ed.). Proceedings of the final coordination meeting, Vienna 24-26 March, 1986. FAO/IAEA, Vienna, Australia, pp 3140.

GANGWAR (A.K.), (K.) ASHOK, (R.K.) SOHANE, (S.) MAHENDRA, (A.) KUMAR, (M.) SINGH. 1998. Influence of rumen $\mathrm{pH}$ on nutrient disappearance of rice straw variety. Indian Journal of Animal Production and Management 14 (1), 5-9.

GOLLUSCIO (R.A.), (J.M.) PARUELO, (J.L.) MERCAU, (V.A.) DEREGI-BUS. 1998. Urea supplemen-tation effects on the utlization of low-quality forage and lamb production in Patagonian rangelands. Grass and Forage Science 53 (1), 47-56.

GRENET (E.), (J.M.) BESLE, 1991. Microbs and fibre degradation. In (J.P.) JOUANY (Ed.). Rumen microbial metabolism and ruminant digestion. INRA (France), 107-130.

GRYSEELS, (G.). 1988. Role of livestock on mixed smallholder farms in the Ethiopian $\mathrm{Hi}$ ghlands : A case study from Baso and Worena wereda near Debre Berhan. Dissertation, Agricultural University, Wageningen, The Netherlands, 249 p.

KANJANAPRUTHIPONG (J.), (R.A.) LENG. 1998. The effect of dietary urea on microbial populations in the rumen of sheep. Asian Australasian Journal of Animal Science 11 (6), 661-672.

KOMISARCZUK (B.), (M.) DURAND. 1991. Effects of mineral on microbial metabolism. In «Rumen microbial metabolism and ruminant digestion » (JOUANYJ P,eds.), pp 179-198, INRA (France). ISBN 2-7380-0345-1.

MADHU (M.), (G.P.) SINGH. 1999. Effect of UMMB on nitrogen parameters in rumen and its utilization in buffaloes fed straw diet. Buffalo Journal 15 (2), 143-152.

MANSO-ALONSO (T.), (T.) CASTROMADRIGAL, (A.) RUIZMANTE-CON, (G.) RODRIGUEZLOPEZ, (A.) FALAGAN-PIETO. 1999. Rumen degradation of shrub com-munities from semi-arid lands in southeast of Spain in two sheep genotypes (Segurena vs Merino). Archivos de Zootecnia 48 (181), 95-100.

MCINTIRE (J.), (D.) BOURZAT, (P.) PINGALI, 1992. Crop-Livestock Interaction in Sub-Saharan Africa. World Bank régional and sectorial studies. The World Bank (Washington D.C., USA), $246 \mathrm{p}$.

MICHALET-DOREAU (B.), (R.) VERITE, (P.) CHAPOUTOT. 1987. Méthodologie de mesure de la dégradabilité in sacco de l'azote des aliments dans le rumen. Bulletin Technique CRVZ Teix 69, 5-7.

NANDRA (K.S.), (A.) HENDRY, (R.C.) DOBOS. 1993. A study of volontary intake and digestibility of roughages in relation to their degradation characteristics and retention time in the rumen. Animal Feed Science and Technology 43, 227-237.

NIANOGO (A.J.), (L.) SOMA, (G.F.X.) BONKOUNGOU, (S) NASSA, (S.J.) ZOUNDI. 1995. Utilisation optimale de la graine de coton et des fourrages locaux pour l'engraissement des ovins Djallonké type Mossi. Revue Réseau Amélioration Production Agricole Milieu Aride, 7, 179-195.

NIANOGO (A.J.), (V.) BOUGOUMA-YAMEOGO, (R.) CORDESSE. 1997. Ingestibilité et digestibilité de deux fourrages tropicaux distribués en l'état, traités à l'urée ou complémentés en matières azotées. Annales de Zootechnie 46, 439-449.

OLSON (K.C.), (R.C.) COCHRAN, (T.J.) JONES, (E.S.) VANZANT, (E.C.) TITGEMEYER, (D.E.) JOHNSON. 1999. The effect of ruminal administration of supplemental degra-dable intake protein and starch on utilization of low-quality warm-season grass hay by beef steers. Journal of Animal Science 4,10161025.

OOSTING (S.J.), (A.) WAANDERS. 1993. The effect of rumen am-monia nitrogen concentration on intake and digestion of wheat straw by goats. Animal Feed Science and Technology 43, 31-40.

ØRKOV (E.R.), (I.) McDONALD. 1979. The estimation of protein degra-dability in the rumen from incubation measurements weighted according to rate of passage. Journal of Agricultural Science Cambridge 92, 499- 
503.

ØRSKOV (E.R.), 1995. Optimising rumen environment for cellulose digestion. In (R.J.) WALLACE, (A.) LAHLOU-KASSI (Ed.). Rumen ecology research planning. Proceedings of a workshop held at ILRI Addis Ababa, Ethiopia. ILRI Nairobi, KENYA, 177182.

OUEDRAOGO (T.), 1998. Contribution à l'étude de l'utilisation digestive comparée des régimes à base de fourrages pauvres chez le mouton et l'âne. Thèse de doctorat de $3^{\text {è }}$ cycle. Université de Ouagadougou, BURKINA FASO,141 p.

PENNING DE VRIES (F.W.T.), (M.A.) DJITEYE, 1982. La productivité des pâturages sahéliens : une étude des sols, des végétations et de l'exploitation de cette ressources naturelle. Ed. PUDOC, Wageningen (The Netherlands), $525 \mathrm{p}$.

PRESTON (T.R.), 1986. Better utilization of crop residues and by-products in animal feeding : Research guidelines : 2 - A practical manual for Research workers. FAO - Rome (Italie), $154 \mathrm{p}$.

RANILLA (M.J.), (M.D.) CARRO, (C.) VALDES, (F.J.) GIRALDEZ, (S.) LOPEZ. 1997. A comparative study of ruminal activity in Churra and Merino sheep offered alfalfa hay. Animal Science 65, 121-128.

RUEDA (E.), (J.) De COMBELLAS. 1999. Effect of multinutrient blocks supplementation within a sheep production system. II. Ruminal parameters and blood urea levels. Revista de la Facultad de Agro-nomia, Universidad del Zulia 16 (1), 89-99.

SANSOUCY (R.). 1995. News developments in the manufacture and utilization of multinutrient blocks. World Animal Review 82 (1), 78-83.

ANONYME 2, 1990 : (SAS). 1990. SAS/STAT User's guide, Volume 2 GLM - VARCOP, Version 6, Fourth edition. SAS Institute Inc., Carry USA, 893-993.

SAVADOGO (M.), 2000. Crop residue management in relation to sustainable land use. A case study in Burkina Faso. Disertation Thesis,
PhD. Wageningen Agri-cultural University, The Nether-lands, $159 \mathrm{p}$.

SAWADOGO (L.L.), (S.J.) ZOUNDI, (A.J.) NIANOGO. 1995. Analyse de quelques caractéristiques du milieu ruminal d'ovins alimentés sur parcours naturels: incidence d'une complémentation azotée sur les niveaux de $\mathrm{N}-\mathrm{NH}_{3}$ et de $\mathrm{pH}$. Agronomie Africaine VII (1), 34-41.

SINGH (P.), (A.K.) VERMA, (R.S.) DASS, (U.R.) MEHRA. 1999. Performance of Pashmina kid goats fed oak (Quercus senecarpifolia) leaves supplemented with a urea molasses mineral block. Small Ruminant Research 31 (3), 239-244.

STRITZLER (N.P.), (B.B.) JENSEN, (J.) WOLTRUP. 1998. Factors affec-ting degradation of barley straw in sacco and microbial activity in the rumen of cows fed fibre-rich diets. III. The amount of suppemental energy. Animal Feed Science and Technology 70, 225238.

THIVEND (P.), (G.) FONTY, (J.P.) JOUANY, (M.) DURAND, (Ph.) GOUET. 1985. Le fermenteur rumen. Reproduction, Nutrition, Développement 25 (4B), 729-753.

TOPPO (S.), (A.K.) VERMA, (R.S.) DASS, (U.R.) MEHRA. 1997. Nutrient utilization and rumen fermentation pattern in crossbred cattle fed different planes mineral block. Animal Feed Science and Technology 64, 101-112.

URBANIAK (M.). 1995. Effects of blood meal, fish meal, soyabean meal or casein on rumen metabolism in lambs. Small Ruminant Research 18, 207-212.

VETH (M.J.), (E.S.) De KOLVER, (D.) COTTLE. 1999. Pasture digestion in response to change in ruminal $\mathrm{pH}$. 59th Conference, Holy Cross College, Mosgiel, 28 June-1st July 1999. Proceedings of the New-Zealand Society of Animal Production 59, 66-69.

ZOUNDI(S.J.), (L.L.) SAWADOGO, (A.J.) NIANOGO. 1994. Croissance compensatrice d'ovins alimentés sur parcours naturels : analyse des gains de poids vif et des caractéristiques de la carcasse. Rev. Rés. Amélior. prod. Agr. Milieu Aride 6 , 179-196. 